\title{
"GAUGING" THE FLUID
}

\author{
Subir Ghosh \\ Physics and Applied Mathematics Unit, \\ Indian Statistical Institute, \\ 203 B. T. Road, Calcutta 700108, \\ India.
}

\begin{abstract}
:
A consistent framework has been put forward to quantize the isentropic, compressible and inviscid fluid model in the Hamiltonian framework, using the Clebsch parameterization. The naive quantization is hampered by the non-canonical (in particular field dependent) Poisson Bracket algebra. To overcome this problem, the Batalin-Tyutin [11] quantization formalism is adopted in which the original system is converted to a local gauge theory and is embedded in a canonical extended phase space. In a different reduced phase space scheme [13] also the original model is converted to a gauge theory and subsequently the two distinct gauge invariant formulations of the fluid model are related explicitly. This strengthens the equivalence between the relativistic membrane (where a gauge invariance is manifest) and the fluid (where the gauge symmetry is hidden). Relativistic generalizations of the extended model is also touched upon.
\end{abstract}

PACS Numbers: 11.15.-q, 47.65.+a

Key Words: gauge theory, BT quantization, fluid. 


\section{Introduction}

Extended objects, a generic example being $d$-branes [1], are receiving more and more attention in high energy physics. The equivalence between membrane $(d=2$ brane) and planar fluid mechanics was revealed some years ago [2]. Subsequently this connection was established in arbitrary dimensions [3]. In fact a specific fluid model, the Chaplygin gas in $(d, 1)$ space-time and the Poincare invariant Born-Infeld model in $(d, 1)$ space-time, both bear a common ancestry to the reparameterization invariant Nambu-Goto $d$-brane theory in $(d+1,1)$ space-time. These issues are discussed in [3, 4].

The concept of symmetry and in particular local gauge invariance has permeated through the present day theoretical physics. In fact, the gauge invariance, (specifically the reparameterization invariance), of the Nambu-Goto theory plays a key role in establishing the above mentioned equivalence, since a choice of light-cone gauge or parameterisation in the NambuGoto action leads to the Chaplygin gas, whereas a Cartesian parameterisation or a combination of the light-cone gauge and a hodographic transformation, (in which the independent and dependent variables are interchanged), yields the Born-Infeld model [3]. These mappings clearly establish the complete integrability of the $d=1$ Chaplygin gas [5] and $d=1$ Born-Infeld model [6] since the parent Nambu-Goto 1-brane (string) moving in a 2-space (plane) is completely integrable.

The Hamiltonian formulation of the inviscid fluid was initiated by the work of Landau [7], who provided the Hamiltonian and the Poisson Bracket algebra of the degrees of freedom, (density and velocity fields), comprising the fluid system. The discussion concerning the Chaplygin gas model pertains to irrotational fluid. (Obviously there can not be any vorticity in one spatial dimension.) The Hamiltonian system for irrotational fluid (in any dimension) becomes very simple, with the velocity field expressible as a gradient of a single scalar degree of freedom (Clebsch variable [8]). However, it has been pointed out in [9] that treated as constraint system in the Dirac Hamiltonian framework [10, the irrotational fluid does not show the gauge invariance, which manifests itself as a $U(1)$ phase invariance in the relativistic membrane. A Batalin-Tyutin (BT) extended space analysis [11] of the above model [12] restores the gauge invariance, albeit in the extended phase space. Subsequently, a connection was established in [9] between the (extended space) gauge invariance [12] and a gauge invariance in the physical sector [9]. The latter result exploits the reduced space scheme discussed in [13]. In this sense the gauge invariance is hidden [9] in the Hamiltonian fluid model.

Let us now put our work in the proper perspective. We do not restrict ourselves to irrotational fluid and instead concentrate on the the general case where the fluid can possess vorticity as well. Essentially, in the next three sections, we have carried out a similar analysis as that of [12, 9] for arbitrary fluid motion. There are qualitative changes since the velocity becomes a non-linear combination three Clebsch variables [14, 15]. This non-linearity makes the BT [11] analysis and reduced space analysis [13] quite involved. Interestingly, we have shown that in the general case of arbitrary fluid motion as well, there exists a mapping between the gauge symmetries in the extended space approach [11] and reduced space scheme [13]. However, the simple $U(1)$ gauge group of the irrotational case is now replaced by the symmetry group of orientation and area preserving diffeomorphism [16].

An earlier work in the context of quantization of fluid system with vortices is the classic paper by Rasetti and Regge [17] where the fluid under consideration is incompressible that is 
of constant density. Since we are dealing with a compressible fluid, results equivalent to that of [17] can be recovered by further constraining our system to a constant density one. We will return to further comparisons between our formalism and that of [17] in Section 3.

The study of the non-relativistic, isentropic, inviscid fluid has emerged lately as an area of intense activity, principally due to its broad applicability. The solutions of the Galilleo invariant system in $d$-dimensions offer solutions for the $d+1$-dimensional relativistic membrane [18]. Also, the subject has identifications with the hydrodynamical description of quantum mechanics [19], parton model [20], hydrodynamics of superfluids [21] and black hole cosmology 22.

The paper is organized as follows: In section 2, we reproduce briefly the conventional Hamiltonian fluid dynamics. Subtleties regarding the introduction of the Clebsch variables are also mentioned. Sections $\mathbf{3}$ and $\mathbf{4}$ deal with the construction of a local gauge theory for the fluid. Specifically, section 3 consists of the BT extension scheme and $\mathbf{4}$ constitutes the reduced space formalism. Relativistic generalizations of the above modified fluid models are also touched up on. The paper ends with a conclusion in $\mathbf{5}$.

\section{Hamiltonian description of the fluid}

Let us start by providing the conventional Hamiltonian formulation of fluid dynamics [3]. We will be considering inviscid, isentropic and compressible fluid in three space dimensions, whose dynamics is governed by the continuity equation and Euler equation,

$$
\partial_{t} \rho+\partial_{i}\left(\rho v^{i}\right)=0, \quad \partial_{t} v_{i}+\left(v^{j} \partial_{j}\right) v_{i}=f_{i},
$$

where $\rho$ and $v_{i}$ denote the density and velocity fields respectively. We keep $f_{i}$ arbitrary for the time being. The above equations of motion (西) are generated by

$$
\partial_{t} \rho(x)=\{\rho(x), H\}, \partial_{t} v_{i}(x)=\left\{v_{i}(x), H\right\}, \quad H\left[\rho, v_{i}\right]=\int d^{3} y \mathcal{H}(y),
$$

using the following Hamiltonian and the (non-canonical) Poisson Bracket (PB) algebra [7]

$$
\begin{gathered}
\mathcal{H}=\frac{1}{2} \rho v_{i} v_{i}+U, \\
\{\rho(x), \rho(y)\}=0, \quad\left\{v_{i}(x), \rho(y)\right\}=\frac{\partial}{\partial x^{i}} \delta(x-y), \quad\left\{v_{i}(x), v_{j}(y)\right\}=-\frac{\partial_{i} v_{j}-\partial_{j} v_{i}}{\rho} \delta(x-y) .
\end{gathered}
$$

Note that we consider only those $f_{i}$ in (1i) which can be generated by some $U$.

It is worth mentioning that generalizations of the $\left\{v_{i}(x), v_{j}(y)\right\} \mathrm{PB}$ in (3) has been discussed in the literature [23], where $\rho$ in the denominator is replaced by $\left(\rho-C_{0}\right), C_{0}$ being a dynamical invariant of the system. Different limiting values for $C_{0}$ lead to distinct regimes of physical interest. The present system with $C_{0}=0$ was derived before for superfluid in zero temperature limit.

However, a canonical Lagrangian formulation of the above is lacking due to the presence of the fluid helicity term,

$$
C=\int d^{3} x\left(\epsilon_{i j k} v^{i} \partial_{j} v^{k}\right)
$$


which, being a Casimir of the theory, creates an obstruction in the inversion of the symplectic matrix [15, 3]. To overcome this problem, albeit in the special case of helicity-less flows, Clebsch parameterization [8] of the velocity field $v_{i}$ and its associated PB algebra are introduced,

$$
\begin{gathered}
v_{i}(x) \equiv \partial_{i} \theta(x)+\alpha(x) \partial_{i} \beta(x), \\
\{\theta(x), \alpha(y)\}=-\frac{\alpha}{\rho} \delta(x-y),\{\beta(x), \alpha(y)\}=\frac{1}{\rho} \delta(x-y),\{\theta(x), \rho(y)\}=\delta(x-y) .
\end{gathered}
$$

These are the only non-zero PBs. This parameterization renders the helicity variable to a surface term without any bulk contribution and obviously reproduces the previous equations of motion. The Lagrangian providing the correct symplectic structure [3] and equations of motion is

$$
\mathcal{L}=\dot{\theta} \rho+\dot{\beta} \alpha \rho-\left(\frac{1}{2} \rho v_{i} v_{i}+U\right)
$$

In our subsequent discussions, we will always use the Clebsch variables.

The non-canonical algebra, posited in (3) or equivalently in (5), is field dependent and hence not conducive for a quantization programme, since the basic Green functions, (i.e. the two-point functions or propagators), can not be defined in a perturbative framework. This motivates us to consider the gauge invariant formulation of the fluid.

\section{Extended space (BT) quantization}

We start by digressing a little on the constraint analysis of Dirac [10] in a Hamiltonian framework. In this scheme, the constraints are termed as First Class Constraints (FCC) if they commute (in the PB sense, modulo constraints) or Second Class Constraints (SCC) if they do not. The FCCs induce gauge invariance in the theory whereas the SCCs tend to modify the symplectic structure of the phase space for compatibility with the SCCs. The above modification induces a replacement of the PBs by Dirac Brackets (DB) [10] as defined below,

$$
\{A(x), B(y)\}_{D B}=\{A(x), B(y)\}-\int\left(d^{3} z d^{3} w\right)\left\{A(x), \eta_{\alpha}(z)\right\}\left\{\eta_{\alpha}(z), \eta_{\beta}(w)\right\}^{-1}\left\{\eta_{\beta}(w), B(y)\right\} .
$$

where $\eta_{\alpha}(x)$ refer to th SCCs.

Primarily, we would like to construct a canonical phase space from which the non-canonical PB algebra can be derived naturally as DBs [24]. This requires an embedding of the original system in a larger phase space having independent and commuting canonical pairs,

$$
\left(\theta, \Pi_{\theta} \equiv \rho\right) ;\left(\alpha, \Pi_{\alpha}\right) ;\left(\beta, \Pi_{\beta}\right),
$$

with $\left\{\theta(x), \Pi_{\theta}(y)\right\}=\delta(x-y)$ etc. Since we have introduced two extra variables in $\Pi_{\alpha}$ and $\Pi_{\beta}$, we also introduce two SCCs [24]

$$
\eta_{1} \equiv \alpha \Pi_{\theta}-\Pi_{\beta} ; \quad \eta_{2} \equiv \Pi_{\alpha} .
$$

In the present case, the SCCs in (9) reproduce (间) as DBs from the above canonical set and also keep the degrees of freedom count same as the original one. 1

\footnotetext{
${ }^{1}$ It should be pointed out that the non-canonical algebra (5) can be reproduced most economically by exploiting the symplectic quantization formalism 3. 25. However, we have pursued the Dirac schme 10] here, keeping in mind the subsequent quantization in the BT formalism [11].
} 
However, in general, the DB formalism [10] can create problems in quantizing the theory since the modified symplectic structure may become field dependent, (if non-linear constraints are present [26]), making it difficult for them to be elevated to quantum commutators via the correspondence principle. With the non-linear constraints (9), precisely this situation is encountered in the present case giving rise to field dependent DBs (5).

To overcome the above mentioned pathology, a systematic procedure has been formulated by Batalin and Tyutin (BT) [1]] whereby one enlarges the phase space, and subsequently the constraints as well as the Hamiltonian accordingly, so that the SCCs turn into FCCs in the enlarged canonical phase space. Quantization of the resulting gauge theory is well understood. Essentially one is trading the original system of physical degrees of freedom having a non-canonical PB (or equivalently DB) structure and (possibly) a simple looking Hamiltonian with an equivalent, extended model having canonical phase space and (possibly) a more complicated looking Hamiltonian. The obvious advantage of the latter over the former is that the BT extended model lives in a canonical phase space and hence is suitable for perturbative quantization. The relevant formulas regarding the BT extension for our purpose are listed below.

Let us consider a generic set of constraints $\left(\Theta_{\alpha}, \Psi_{l}\right)$ and a Hamiltonian operator $H$ with the following PB relations,

$$
\begin{gathered}
\left\{\Theta_{\alpha}(q), \Theta_{\beta}(q)\right\} \approx \Delta_{\alpha \beta}^{\mu \nu}(q) \neq 0 ;\left\{\Theta_{\alpha}(q), \Psi_{l}(q)\right\} \approx 0 \\
\left\{\Psi_{l}(q), \Psi_{n}(q)\right\} \approx 0 ; \quad\left\{\Psi_{l}(q), H(q)\right\} \approx 0 .
\end{gathered}
$$

In the above $(q)$ collectively refers to the set of variables present prior to the BT extension and " $\approx$ " means that the equality holds on the constraint surface. Clearly $\Theta_{\alpha}$ and $\Psi_{l}$ are SCC and FCC [10] respectively.

In systems with non-linear SCCs, (such as the present one), in general the DBs can become dynamical variable dependent [26] due to the $\left\{A, \Theta_{\alpha}\right\}$ and $\Delta_{\alpha \beta}$ terms, leading to problems for the quantization programme. To cure this type of pathology, BT formalism is a systematic framework where one introduces the BT variables $\phi_{a}^{\alpha}$, obeying

$$
\left\{\phi^{\alpha}, \phi^{\beta}\right\}=\omega^{\alpha \beta}=-\omega^{\beta \alpha}
$$

where $\omega^{\alpha \beta}$ is a constant (or at most a c-number function) matrix, with the aim of modifying the SCC $\Theta_{\alpha}(q)$ to $\tilde{\Theta}_{\alpha}\left(q, \phi^{\alpha}\right)$ such that,

$$
\left\{\tilde{\Theta}_{\alpha}(q, \phi), \tilde{\Theta}_{\beta}(q, \phi)\right\}=0 ; \tilde{\Theta}_{\alpha}(q, \phi)=\Theta_{\alpha}(q)+\Sigma_{n=1}^{\infty} \tilde{\Theta}_{\alpha}^{(n)}(q, \phi) ; \tilde{\Theta}^{(n)} \approx O\left(\phi^{n}\right)
$$

This means that $\tilde{\Theta}_{\alpha}$ are now FCCs and in particular abelian [11]. The explicit terms in the above expansion are [1],

$$
\begin{gathered}
\tilde{\Theta}_{\alpha}^{(1)}=X_{\alpha \beta} \phi^{\beta} ; \Delta_{\alpha \beta}+X_{\alpha \gamma} \omega^{\gamma \delta} X_{\beta \delta}=0 \\
\tilde{\Theta}_{\alpha}^{(n+1)}=-\frac{1}{n+2} \phi^{\delta} \omega_{\delta \gamma} X^{\gamma \beta} B_{\beta \alpha}^{(n)} ; n \geq 1 \\
B_{\beta \alpha}^{(1)}=\left\{\tilde{\Theta}_{\beta}^{(0)}, \tilde{\Theta}_{\alpha}^{(1)}\right\}_{(q)}-\left\{\tilde{\Theta}_{\alpha}^{(0)}, \tilde{\Theta}_{\beta}^{u(1)}\right\}_{(q)}
\end{gathered}
$$




$$
B_{\beta \alpha}^{(n)}=\sum_{m=0}^{n}\left\{\tilde{\Theta}_{\beta}^{(n-m)}, \tilde{\Theta}_{\alpha}^{(m)}\right\}_{(q, p)}+\sum_{m=0}^{n}\left\{\tilde{\Theta}_{\beta}^{(n-m)}, \tilde{\Theta}_{\alpha}^{(m+2)}\right\}_{(\phi)} ; n \geq 2
$$

In the above, we have defined,

$$
X_{\alpha \beta} X^{\beta \gamma}=\omega_{\alpha \beta} \omega^{\beta \gamma}=\delta_{\alpha}^{\gamma} \delta
$$

A very useful idea is to introduce the improved function $\tilde{f}(q)$ [11] corresponding to each $f(q)$,

$$
\begin{gathered}
\tilde{f}(q, \phi) \equiv f(\tilde{q})=f(q)+\Sigma_{n=1}^{\infty} \tilde{f}(q, \phi)^{(n)} ; \tilde{f}^{(1)}=-\phi^{\beta} \omega_{\beta \gamma} X^{\gamma \delta}\left\{\Theta_{\delta}, f\right\}_{(q)} \\
\tilde{f}^{(n+1)}=-\frac{1}{n+1} \phi^{\beta} \omega_{\beta \gamma} X^{\gamma \delta} G(f)_{\delta}^{\lambda(n)} ; n \geq 1 \\
G(f)_{\beta}^{(n)}=\sum_{m=0}^{n}\left\{\tilde{\Theta}_{\beta}^{(n-m)}, \tilde{f}^{(m)}\right\}_{(q)}+\Sigma_{m=0}^{(n-2)}\left\{\tilde{\Theta}_{\beta}^{(n-m)}, \tilde{f}^{(m+2)}\right\}_{(\phi)}+\left\{\tilde{\Theta}_{\beta}^{(n+1)}, \tilde{f}^{(1)}\right\}_{(\phi)}
\end{gathered}
$$

which have the property $\left\{\tilde{\Theta}_{\alpha}(q, \phi), \tilde{f}(q, \phi)\right\}=0$. Thus the improved functions are FC or equivalently gauge invariant. The subscript $(\phi)$ and $(q)$ in the PBs indicate the improved variables with respect to which the PBs are to be taken. It can be proved that extensions of the original FCC $\Psi_{l}$ and Hamiltonian $H$ are simply,

$$
\tilde{\Psi}_{l}=\Psi(\tilde{q}) ; \quad \tilde{H}=H(\tilde{q}) .
$$

One can also reexpress the converted SCCs as $\tilde{\Theta}_{\alpha}^{\mu} \equiv \Theta_{\alpha}^{\mu}(\tilde{q})$. The following identification theorem holds,

$$
\{\tilde{A}, \tilde{B}\}=\{A, \tilde{B}\}_{D B} \quad ;\left.\quad\{\tilde{A}, \tilde{B}\}\right|_{\phi=0}=\{A, B\}_{D B} ; \quad \tilde{0}=0 .
$$

Hence the outcome of the BT extension is the closed system of FCCs with the FC Hamiltonian given below,

$$
\left\{\tilde{\Theta}_{\alpha}^{\mu}, \tilde{\Theta}_{\beta}^{\nu}\right\}=\left\{\tilde{\Theta}_{\alpha}^{\mu}, \tilde{\Psi}_{l}\right\}=\left\{\tilde{\Theta}_{\alpha}^{\mu}, \tilde{H}\right\}=0 ;\left\{\tilde{\Psi}_{l}, \tilde{\Psi}_{n}\right\} \approx 0 ;\left\{\tilde{\Psi}_{l}, \tilde{H}\right\} \approx 0 .
$$

In general, due to the non-linearity in the SCCs, the extensions in the improved variables, (and subsequently in the FCCs and FC Hamiltonian), may turn out to be infinite series. This type of situation has been encountered before [26]. Fortunately this complication does not surface here. The are no FCCs in our system, (analogous to $\Psi_{l}$ ) and identifying the SCCs $\eta_{\alpha}$ in (9) in the present case with $\Theta_{\alpha}$ in (10), we compute the BT extended FCCs as,

$$
\tilde{\eta}_{1} \equiv \eta_{1}+\phi_{1} ; \quad \tilde{\eta}_{2} \equiv \eta_{2}-\Pi_{\theta} \phi_{2},\left\{\tilde{\eta}_{1}, \tilde{\eta}_{2}\right\}=0
$$

The BT fields obey $\left\{\phi_{1}(x), \phi_{2}(y)\right\}=\delta(x-y)$. To ensure that there are no further constraints, we need a Hamiltonian that commutes with the FCCs. The following variables,

$$
\tilde{\theta}=\theta+\alpha \phi_{2}, \tilde{\Pi}_{\theta}=\Pi_{\theta}, \tilde{\alpha}=\alpha+\frac{\phi_{1}}{\Pi_{\theta}}, \tilde{\Pi}_{\alpha}=\Pi_{\alpha}-\Pi_{\theta} \phi_{2}, \tilde{\beta}=\beta-\phi_{2}, \tilde{\Pi}_{\beta}=\Pi_{\beta}, \tilde{\phi}_{i}=0
$$


are gauge invariant [11] in the sense that they commute with the FCCs. Hence all quantities written in terms of the redefined variables are gauge invariant in the extended space. In particular, the modified (free) Hamiltonian reads

$$
\left.\tilde{\mathcal{H}}\right|_{\text {free }}=\frac{1}{2}\left(\tilde{\Pi}_{\theta} \tilde{v}_{i} \tilde{v}_{i}\right)=\frac{1}{2} \Pi_{\theta}\left[\partial_{i}\left(\theta+\alpha \phi_{2}\right)+\left(\alpha+\frac{\phi_{1}}{\Pi_{\theta}}\right) \partial_{i}\left(\beta-\phi_{2}\right)\right]^{2} .
$$

The remaining interaction terms in $H$, if present, will also be extended in a similar way. This Hamiltonian (26) together with the FCCs (24) and the canonical phase space is the gauge invariant system we were looking for. This constitutes one of the major results of the paper.

It may be worthwhile at this point to comment on the apparently involved structure of the extended Hamiltonian (26) with a dynamical variable residing in the denominator. As has been emphasized in the Introduction, this complexity is unavoidable. However, even then, this extended system together with its canonical phase space is more suitable for quantization in a perturbative framework. For example, for "small" or "large" $\Pi_{\theta}$, i.e. density, in some scale, one can expand $\Pi_{\theta}$ around a background or expand in terms of $\left(1-\Pi_{\theta}\right)$ in the offending term in (26).

It will be appropriate to make a comparison between [17] and our analysis. Essentially the aim of Hamiltonian formulation of a dynamical system is to provide a Hamiltonian function and a set of PBs, which will reproduce (via the Hamiltons equations of motion) a given set of equations of motion of the system. Indeed, there are some consistency conditions for the PBs (such as Jacobi identity etc.). Now, the basic set of dynamical variables in [17] are different from our set, although it is obvious that there exists a mapping between the two sets, once the additional constraint of constant density is imposed on our model. But more importantly, it should be noted that the basic quantum commutation relations in [17] are operator valued and hence perturbative computations will be difficult to perform. On the other hand, the present system is embedded in the BT extended space, which is completly canonical (i.e. not operator valued) by construction! and thus is amenable to conventional perturbative analysis. Because of the introduction of auxiliary fields a standard BRST [27] quantization is to be performed, which has not been carried through here.

To make contact with the physical system, another alternative is to reduce the dimension of the BT extended phase space by additional gauge fixing constraints, (two in this case, $\tilde{\eta}_{3}$ and $\tilde{\eta}_{4}$, corresponding to two FCCs), with the only restriction that $\tilde{\eta}_{a}, a=1, . ., 4$ constitute an SCC system that is $\operatorname{det}\left|\left\{\tilde{\eta}_{a}, \tilde{\eta}_{b}\right\}\right| \neq 0$. A consistency check is to see that the original system is recovered in the so called unitary gauge, $\tilde{\eta}_{3} \equiv \phi_{1} \approx 0, \tilde{\eta}_{4} \equiv \phi_{2} \approx 0$. It is interesting to note that in the extended space, the Hamiltonian or any other observable can, in general, depend upon the Clebsch variables, which are not expressible in terms of the velocity $v_{i}$. However, it should be remembered that they describe physically allowed systems since they are gauge equivalent to the physical system.

It might be convenient, (although not necessary), to consider the gauges of the form $\tilde{\eta}_{3} \equiv$ $\phi_{1}-F, \tilde{\eta}_{4} \equiv \phi_{2}-G$, to remove the BT fields directly. $F$ and $G$ can contain the physical fields as well. For a particular gauge, one has to construct the corresponding $D B$ s and compute the equations of motion using the $D B \mathrm{~s}$ in reduced phase space, where the SCCs have been used strongly. Once again, the degrees of freedom count agrees with the original one. Consider the special class of gauge transformations: $\phi_{1}=0, \phi_{2}=$ constant. These will not change the $\left(v_{i}, \rho\right)$ algebra. Hence they can be identified as the conventional canonical transformations. 
Furthermore, additional constraints, such as incompressibility [24], can be included in this setup in the form $\rho=$ constant, which under time persistence generates another constraint $\partial_{i} \tilde{v}_{i}$. This SCC pair leads to [24].

The constants of motion for the free theory are obviously the energy $\tilde{H}$, the momenta $\tilde{P}_{i}=\int\left(\rho \partial_{i} \theta+\Pi_{\alpha} \partial_{i} \alpha+\Pi_{\beta} \partial_{i} \beta+\phi_{2} \partial_{i} \phi_{1}\right)$, the angular momenta $\tilde{L}^{i j}=\int\left(r^{i} \tilde{\mathcal{P}}^{j}-r^{j} \tilde{\mathcal{P}}^{i}\right)$ and the boost generator $\tilde{B}^{i}=t \tilde{P}_{i}-\int\left(r_{i} \rho\right)$, effecting the transformation

$$
\left\{\tilde{v}_{i}, u_{j} \tilde{B}_{j}\right\}=-t\left(u_{j} \partial_{j}\right) \tilde{v}_{i}+u_{i},\left\{\rho, u_{j} \tilde{B}_{j}\right\}=-t\left(u_{j} \partial_{j}\right) \rho
$$

Obtaining the Lagrangian is indeed straightforward. The first order form is

$$
\begin{gathered}
\mathcal{L}=\Pi_{\theta} \dot{\theta}+\Pi_{\alpha} \dot{\alpha}+\Pi_{\beta} \dot{\beta}+\phi_{2} \dot{\phi}_{1}-\tilde{\mathcal{H}}-\lambda_{1} \tilde{\eta_{1}}-\lambda_{2} \tilde{\eta}_{2} \\
\equiv \Pi_{\theta} \dot{\theta}+\phi_{2} \dot{\phi}_{1}+\dot{\beta}\left(\alpha \Pi_{\theta}+\phi_{1}\right)+\dot{\alpha} \Pi_{\theta} \phi_{2}-\tilde{\mathcal{H}}-\lambda_{1} \tilde{\eta}_{1}-\lambda_{2} \tilde{\eta}_{2},
\end{gathered}
$$

where $\lambda_{1}$ and $\lambda_{2}$ are multiplier fields and some of the variables have been removed using the equations of motion. The generic gauge transformation is defined as

$$
\delta A=\left\{\int(\epsilon \Psi), A\right\},
$$

where $A, \epsilon$ and $\Psi$ represent some operator, infinitesimal gauge transformation parameter and an FCC respectively. At this stage, one can check explicitly that (27) is invariant under the following two independent sets of gauge transformations corresponding to the two FCCs,

$$
\begin{gathered}
\tilde{\eta}_{1} \rightarrow \delta_{1} \Pi_{\theta}=0, \delta_{1} \theta=-\alpha \psi_{1}, \delta_{1} \beta=\psi_{1}, \delta_{1} \alpha=0, \delta_{1} \phi_{1}=0, \delta_{1} \phi_{2}=\psi_{1} ; \\
\tilde{\eta}_{2} \rightarrow \delta_{2} \Pi_{\theta}=0, \delta_{2} \theta=\phi_{2} \psi_{2}, \delta_{2} \beta=0, \delta_{2} \alpha=-\psi_{2}, \delta_{2} \phi_{1}=\Pi_{\theta} \psi_{2}, \delta_{2} \phi_{2}=0,
\end{gathered}
$$

where $\psi_{1}$ and $\psi_{2}$ are gauge transformation parameter functions. Naively taking the unitary gauge, i.e. $\phi_{1}=\phi_{2}=0$, we can recover the Lagrangian posited in [3].

We now discuss briefly the relativistic generalization of the parent free theory. In the relativistic generalization of the free theory [3], the Lagrangian is expressed as

$$
\begin{gathered}
\mathcal{L}_{r e l}=j^{\mu} a_{\mu}-\left(j^{\mu} j_{\mu}\right)^{\frac{1}{2}}, \\
a_{\mu}=\partial_{\mu} \theta+\alpha \partial_{\mu} \beta, j^{\mu}=\left(\rho, \rho v^{i}\right) .
\end{gathered}
$$

Notice that the symplectic structure does not change from the non-relativistic one. Expansion of the square root as

$$
\rho\left(1+v^{i} v_{i}\right)^{\frac{1}{2}} \approx \rho\left(1+\frac{1}{2} v^{i} v_{i}+\ldots\right),
$$

and dropping the uninteresting $\int \rho$ term [3], (since it can only influence the time evolution of $\theta$ by a constant translation), we can recover the non-relativistic Lagrangian in (6) with $U=0$. The Hamiltonian now is modified to

$$
\mathcal{H}_{r e l}=\rho\left[v_{i} v_{i}+\left(1-v_{i} v_{i}\right)^{\frac{1}{2}}\right]
$$

which changes the equations of motion to the following:

$$
\dot{\alpha}=L_{i} \partial_{i} \alpha \quad ; \quad \dot{\beta}=L_{i} \partial_{i} \beta \quad ; \quad \dot{\theta}=-L_{i} \alpha \partial_{i} \beta+\left[v_{i} v_{i}+\left(1-v_{i} v_{i}\right)^{\frac{1}{2}}\right] ; \dot{\rho}=\partial_{i}\left(\rho L_{i}\right),
$$




$$
L_{i}=\frac{1}{\rho} \frac{\partial \mathcal{H}_{r e l}}{\partial v_{i}}=v_{i}\left[2-\left(1-v_{i} v_{i}\right)^{-\frac{1}{2}}\right] .
$$

Notice that in the lowest order, $L_{i} \approx v_{i}+O\left(v^{3}\right)$, the previous equations are recovered.

BT extension of the relativistic model is straightforward since the symplectic structure remains unchanged from the non-relativistic one. One only has to replace the original variables by their gauge invariant counterpart (25) in the covariant expressions (30) and (31).

We briefly remark on the canonical quantization of the BT extended fluid model. Indeed, the BT extension has rendered the phase space canonical and the classical variables are simply elevated to quantum operators with the generic equal time commutators being,

$$
\left[\phi(x), \pi_{\phi}(y)\right]=i \hbar \delta(x-y) .
$$

The FCCs (24,9) are taken in to consideration by invoking the Dirac quantization prescription [10], in which the physical states are defined as [28, 29]

$$
\mid \text { Ph State }>\equiv \delta\left(\tilde{\eta}_{1}\right) \delta\left(\tilde{\eta}_{2}\right) \mid \text { State }>\text {, }
$$

indicating that the physical states are annihilated by the FCCs. One can immedietly get some idea on the qualitative nature of the (lowest order) quantum corrections involved, say in the energy spectrum, by sandwitching the extended space FC Hamiltonian operator (26) between the physical states as defined above. Following [28, 29] we can resort to Weyl ordering the quantum operator products and for simplicity let us exploit the unitary gauge. Naively it might seem that there can appear non-trivial quantum corrections. However it is straightforward to check that the structure of the constraints in the unitary gauge and the derivative operators involved (in the Hamiltonian) conspire to produce the quantum corrections in the form of total derivatives only, which are assumed to vanish. Notice that in an apparantly similar circumstance in [29], in the $C P^{1}$-model with Hopf interaction, non-zero quantum corrections appeared in the form of total derivatives in $\mathrm{t}$ ! he topologically non-trivial sector only. No such topological protection is present here.

\section{Reduced space quantization}

As mentioned in the Introduction, we now discuss briefly the alternative scheme [13] of inducing gauge invariance in a system subjected to SCCs only, without enlarging the phase space, that is no BT fields are introduced. This prescription is particularly suitable for the special case of only two SCCs, as is the case here, (9). The primary idea is to consider (a suitably modified form of) one of the SCCs as the only FCC and discard the other SCC, which may be thought of as a particular gauge fixing condition one is free ignore. There is a prescription [13] by which gauge invariant counterparts of all the variables, (and hence the Hamiltonian and other relevant quantities as well), can be constructed. In fact, this formalism and the BT extended scheme can be related in a formal way, at least for two SCCs [13], which however will not be discussed here. For two generic SCCs $Q_{1}$ and $Q_{2}$, with $\left\{Q_{1}, Q_{2}\right\}=\Lambda \neq 0$, the SCC pair can be replaced by a single FCC $\chi=\Lambda^{-1} Q_{1}$ with $Q_{2}$ dropped. Obviously taking $Q_{2}$ instead of $Q_{1}$ in $\chi$ is also a valid choice. It can be checked that for any degree of freedom $A,\{\chi, \tilde{A}\}=0$, where $\tilde{A}$, defined below, is the gauge invariant counterpart of $A$,

$$
\tilde{A}=A-Q_{2}\{\chi, A\}+\frac{1}{2 !} Q_{2}^{2}\{\chi,\{\chi, A\}\}-\frac{1}{3 !} Q_{2}^{3}\{\chi,\{\chi,\{\chi, A\}\}\}+\ldots
$$


In the present case, with the SCC system (9) the gauge invariant fluid models are Case I:

$$
\begin{gathered}
\chi=\frac{\Pi_{\alpha}}{\Pi_{\theta}}, \\
\tilde{\theta}=\theta+\left(\alpha \Pi_{\theta}-\Pi_{\beta}\right) \frac{\Pi_{\alpha}}{\Pi_{\theta}^{2}}, \quad \tilde{\alpha}=\frac{\Pi_{\beta}}{\Pi_{\theta}} .
\end{gathered}
$$

The gauge variation of the first order Lagrangian also vanishes,

$$
\begin{aligned}
\delta \mathcal{L}=\left\{\int d^{3} x(\lambda \chi)\right. & \left.,\left(\Pi_{\theta} \dot{\theta}+\Pi_{\alpha} \dot{\alpha}+\Pi_{\beta} \dot{\beta}\right)-\tilde{H}\right\} \\
& =\lambda \dot{\chi} \approx 0 .
\end{aligned}
$$

Case II:

$$
\begin{gathered}
\chi=\alpha-\frac{\Pi_{\beta}}{\Pi_{\theta}} \\
\tilde{\theta}=\theta+\frac{\Pi_{\alpha} \Pi_{\beta}}{\Pi_{\theta}^{2}}, \quad \tilde{\beta}=\beta-\frac{\Pi_{\alpha}}{\Pi_{\theta}} .
\end{gathered}
$$

The gauge variation of the first order Lagrangian again vanishes,

$$
\delta \mathcal{L}=\lambda \dot{\chi} \approx 0 .
$$

Let us now make a direct contact between the gauge invariant theories formulated in the BT extended scheme in section $\mathbf{3}$ and in the present section. Obviously, the former is the more general one as it contains two FCCs whereas the latter has a single FCC. Considering the FCC of case I above in the reduced space scheme, the gauge transformations are,

$$
\delta \alpha=-\frac{\epsilon}{\Pi_{\theta}}, \quad \delta \theta=\epsilon \frac{\Pi_{\alpha}}{\Pi_{\theta}^{2}} .
$$

Now in the BT formulation, choose a gauge fixing condition as

$$
\mathcal{G} \equiv \phi_{2}-\frac{\Pi_{\alpha}}{\Pi_{\theta}^{2}}
$$

This gauge will render the pair $\left(\mathcal{G}, \tilde{\eta}_{1}\right)$ second class while keeping the first class nature of $\tilde{\eta}_{2}$ intact. Presently, computing DBs with respect to the above SCC pair and using them to compute the gauge variations, one finds that on the FCC surface $\tilde{\eta}_{2} \approx 0$ the gauge transformations in the BT and reduced schemes are identical. For a different gauge condition the FCC in case II above in the reduced scheme can also be matched to the BT results. This verification is the analogue of [9] for a general fluid. This concludes our analysis of the quantization problem of the Hamiltonian fluid model both from an extended space and reduced space points of view. The hidden gauge invariance of the fluid system has also been revealed in both the BT and reduced schemes. Indeed, the above correspondence between the results obtained in the two distinct methods is important as it connects the gauge invariance in the enlarged (BT) space to the one in physical space. 


\section{Discussions}

The Hamiltonian formulation of isentropic, inviscid fluid in three spatial dimensions has been studied as a prelude to its quantization. The Poisson bracket structure, yielding the fluid equations of motion, is non-canonical (in particular field dependent). This creates problem for the convenional quantization programme since the quantum commutators (via the correspondence principle) will also aquire a field dependence and as a result even the propagators can not be defined properly.

This motivates us to exploit the Batalin-Tyutin quantization scheme [11] in which the constraints are taken in account in a canonical extended phase space. The system is also converted to a gauge theory. A second method, the reduced space scheme [13] is also applied to the fluid model. Here also the fluid model is converted to a gauge theory but contrary to the previous framework [11], the phase space extension is not required. This hidden gauge invariance in the fluid model in the latter formulation has also been explicitly connected to the former one. This hidden gauge symmetry actually corresponds to the gauge invariance present in the equivalent relativistic membrane theory. This is a generalization of the works of [9, 12].

The presence of gauge invariance offers more freedom in the analysis of a theory and apparently different models can become identified as gauge equivalent ones, so that results obtained in one model can be carried to the other one. For instance, generally one considers the fluid

system, in physical situation, as being subjected to a pressure term $\left(\frac{\partial_{i} p(x)}{\rho}\right)$ and a constant force such as gravity. By a suitable choice of (translation symmetry breaking) gauge, one can generate these terms in the Euler equation. However, even in the linear approximation, there will be additional terms in the Euler equation besides the above ones and the continuity equation will also be modified. One can say that this set is gauge equivalent to the free theory since the latter is reproduced in the unitary gauge. Indeed. one can trade one type of interaction and source with another one by exploiting the BT gauge equivalence and one set might be better suited to simulate experimentally or analyze theoretically.

The Batalin-Tyutin construction is primarily aimed at providing a canonical framework for quantizing the fluid system. It will indeed be interesting to study the quantized version of the above model presented here in more detail.

Furthermore, gauge invariant interactions of the form $\epsilon_{\mu \nu \sigma \lambda} F^{\mu \nu} \theta \partial^{\sigma} \alpha \partial^{\lambda} \beta$ can also be introduced in the action which will alter the symplectic structure and the resulting theory can be studied perturbativly.

Acknowledgements: I thank Dr. K. Kumar for numerous stimulating discussions. 


\section{References}

[1] For a review see A.Giveon and D.Kutasov, Rev.Mod.Phys. 71 983(1999).

[2] J.Goldstone, (unpublished); M.Bordemann and J.Hoppe, Phys.Lett. B317 315(1993).

[3] For a review, see R.Jackiw, physics/0010042.

[4] R.Jackiw and A.P.Polychronakos, hep-th/9809123; D.Bazeia and R.Jackiw, Ann.Phys.(NY), (hep-th/9803165); R.Jackiw and A.P.Polycronakos, Comm.Math.Phys. 207 107(1999), D.Bazia, Phys.Rev. D59 085007(1999).

[5] I.Landau and E.Lifshitz, Fluid Mechanics, (2nd ed., Pergamon, Oxford, UK, 1987); P.Olver and Y.Nutku, J.Math.Phys. 29 1610(1988).

[6] B.Barbishov and N.Chernikov, Sov.Phys. JETP 24 1371(1967).

[7] L.D.Landau, ZETF 11 592(1941) [English translation: J.Phys.USSR 5 71(1941)].

[8] H.Lamb, Hydrodynamics, (Cambridge University Press, Cambridge, UK, 1932); For subtleties involved in Clebsch parametrization, see S.Deser, R.Jackiw and A.Polychronakos, Phys.Lett.A 279 151(2001).

[9] C.Neves and C.Wotzasek, hep-th/0105281.

[10] P.A.M.Dirac, Lectures on Quantum Mechanics, (Yeshiva University Press, New York, 1964).

[11] I.A.Batalin and I.V.Tyutin, Int.J.Mod.Phys. A6 3255(1992).

[12] C.P.Natividade and H.Boschi-Filho, Phys.Rev.D62 025016 (2000).

[13] P.Mitra and R.Rajaraman, Ann.Phys. (NY) 203 157(1990); R.Anishetty and A.S.Vytheeswaran, J.Phys.A 26, 5613(1993).

[14] A.Thellung, Physica 29 227(2953).

[15] R.Salmon, Ann.Rev.Fluid Mech. 20 225(1988).

[16] S.Bergliaffa, K.Hibbard, M.Stone and M.Vissar, arXiv:cond-mat/0106255.

[17] M.Rasetti and T.Regge, Physica 80A 217(1975).

[18] See the papers by Bazeia and Jackiw in [4].

[19] E.Madelung, Z.Phys. 40 322(1926); E.Merzbacher, Quantum Mechanics, (3rd ed., Wiley, New York, 1998).

[20] A.Jevicki, Phys.Rev. D57 5955(1998).

[21] A.M.J.Shakel. Mod.Phys.Lett.B 4 927(1990). 
[22] A.Yu.Kamenshchik, U.Moschella and V.Pasquier, Phys.Lett.B 487 7(2000).

[23] I.E.Dzyaloshinskii and G.E.Volovick, Ann.Phys. 125 67(1980); G.E.Volovick and V.S.Dotsenko Jr., JETP Lett. 29 576(1979); A.F.Andreev and M.Yu.Kagan, Zh.Eksp.Teor.Fiz. 86 546(1984) [Sov.Phys. JETP 59 318(1984)]; G.E.Volovick, JETP Lett. 64 845(1996).

[24] S.Nguyen and L.A.Tursky, Physica A290 431; ibid A272 48(1999). ibid 57 432(1972); S.Ghosh, arXiv hep-th/0106166.

[25] L.Faddeev and R.Jackiw, Phys.Rev.Lett. 60 1692(1988).

[26] See for example N.Banerjee, R.Banerjee and S.Ghosh, Nucl.Phys. B427 257(1994).

[27] C.Becchi, A.Rouet and R.Stora, Phys.Lett. 52B 344(1979); I.V.Tyutin, Lebedev Preprint FIAN, No. 39 (1975).

[28] W.Oliveira and J.A.Neto, Int.J.Mod.Phys. A12 4895(1997).

[29] B.Chakraborty, S.Ghosh and P.P.Malik, Nucl.Phys. B600 351(2001). 